Original Research Paper

\title{
Is RNA Binding in the Rossmann Fold Essential for GAPDH Intranuclear Functions?
}

\author{
Natalia Krynetskaia, Manali Phadke and Evgeny Krynetskiy \\ Temple University School of Pharmacy, Philadelphia, PA 19140 USA
}

Article history

Received: 11-02-2016

Revised: 03-06-2016

Accepted: 13-06-2016

Corresponding Author:

Evgeny Krynetskiy

Temple University School of

Pharmacy, Philadelphia, PA

19140 USA

Tell: +1 2157074257

Fax: +12157075620

Email:ekrynets@temple.edu

\begin{abstract}
In addition to its prominent enzymatic activity, GAPDH is an enigmatic component of multiple unrelated biochemical entities. In this study, we explored a series of mutated GAPDH and fusion EGFP-GAPDH polypeptides and compared nuclear accumulation, intranuclear mobility and RNA binding properties of the wild type and variant GAPDH proteins. Our results revealed that RNA binding to T99I-mutated GAPDH with nonfunctional $\mathrm{NAD}^{+}$binding center occurred outside the Rossmann fold. At the cellular level, wild type and mutated EGFP-GAPDH demonstrated distinct intranuclear localization in unstressed cells versus cells exposed to genotoxic stress. Wild type EGFP-GAPDH protein localized in the cytoplasm of untreated cells and accumulated in the nucleus following araC treatment $(11.8 \pm 2.72 \%$ vs. $27.4 \pm 4.28 \%$ nuclear EGFP-GAPDH, \% of total EGFP-GAPDH, $\mathrm{p}=0.0007)$. Mutated T99I EGFP-GAPDH accumulated at high level in the nuclei of untreated and araC-treated cells $(34.3 \pm 8.49 \%$ vs. $41.3 \pm 16.0 \%$ nuclear EGFP-GAPDH, $\%$ of total EGFP-GAPDH, $\mathrm{p}=0.21$ ). Mutated T99I EGFP-GAPDH lost its ability to form tight interactions with intranuclear macromolecules. After araC treatment, immobile fraction (1Mf) of wild type EGFP-GAPDH in the nuclei of SW48-297 cells was three times higher $(0.75 \pm 0.127$ vs. $0.26 \pm 0.133 \%, \mathrm{p}<0.0001)$, recovery half-time was three times higher $(0.84 \pm 0.075$ vs. $0.3 \pm 0.085 \mathrm{~s}, \mathrm{p}<0.0001)$ and diffusion coefficient $\mathrm{D}$ was five times lower $(4.6 \pm 0.85$ vs. $23.3 \pm 13.79$ $\left.\mu \mathrm{m}^{2} / \mathrm{s}, \mathrm{p}=0.0001\right)$ compared to cells expressing T99I variant. Our results suggest that the switch between RNA and $\mathrm{NAD}^{+}$binding to GAPDH could be a regulatory mechanism governing participation of GAPDH in $\mathrm{NAD}^{+}-$ dependent complexes and could serve a depot to supply diverse biochemical processes with $\mathrm{NAD}^{+}$.
\end{abstract}

Keywords: Glyceraldehyde-3-Phosphate Dehydrogenase, NAD ${ }^{+}$, Fluorescence Recovery after Photobleaching, Nuclear Proteins, Mutation, RNA

\section{Introduction}

Propensity of Glyceraldehyde 3-Phosphate Dehydrogenase (GAPDH) to bind single-stranded polynucleotides is an intriguing phenomenon which significance remains enigmatic. Three types of polynucleotide-GAPDH interaction have been described, so far: (i) GAPDH binding to RNA, mostly represented by AU-Rich Elements (ARE) in the 3'UTR of mRNA (Nagy and Rigby, 1995; Seidler, 2013) (ii) formation of multiprotein-RNA complexes, with GAPDH and RNA being important structural components (Mazurek et al., 1996; Carlile et al., 1998) (iii) GAPDH binding to single-stranded DNA at telomeric sequences near the chromosomal ends (Sundararaj et al., 2004; Demarse et al., 2009). GAPDHRNA binding affects mRNA stability and template activity (Bonafe et al., 2005; Rodriguez-Pascual et al., 2008; Backlund et al., 2009; Kondo et al., 2011; Ikeda et al., 2012; Zeng et al., 2014). RNA binding activity of GAPDH is attributed to the Rossmann fold which forms $\mathrm{NAD}^{+}$-binding center located at the Nterminus of GAPDH polypeptide (Rossman, 1981). Several lines of evidence including V8 protease digestion, competitive binding and site-mutagenesis experiments support this hypothesis (Nagy and Rigby, 
1995; Sundararaj et al., 2004; Demarse et al., 2009). $\mathrm{NAD}^{+}$which is a co-factor of GAPDH, bears structural resemblance to AU-Rich Elements (ARE) often found in 3'-UTR regions of mRNA (Seidler, 2013). Affinity of GAPDH to ARE results in GAPDH binding to several mRNA coding for c-myc, CSF-1/2, IFN- $\gamma$, IL-2, ET1, AT1R and COX-2 (Nagy and Rigby, 1995; Rodriguez-Pascual et al., 2008; Zhou et al., 2008; Backlund et al., 2009; Ikeda et al., 2012). Importantly, mutagenesis of residues localized at the GAPDH dimer interface impaired binding of GAPDH to ARE within TNF- $\alpha$ mRNA (White et al., 2015).

Our previous studies demonstrated the loss of glycolytic activity in GAPDH variants containing mutations in the Rossmann fold due to dramatically decreased $\mathrm{NAD}^{+}$binding. In this study, we used sitemutagenesis to demonstrate that T99I mutation within the Rossmann fold disrupted $\mathrm{NAD}^{+}$binding but not RNA binding. Our findings indicate that RNA interacts with T99I protein at a site different from that in a wild type GAPDH. The mutant GAPDH also failed to participate in intranuclear interactions in HCT116 colorectal carcinoma cells, as revealed by Fluorescence Recovery After Photobleaching (FRAP) experiments (Phadke et al., 2015). To investigate the relation between GAPDH/RNA-binding activity and dynamic properties of nuclear GAPDH, in this study we compared RNA binding properties, nuclear accumulation and intranuclear mobility of the wild type and T99I GAPDH mutant variant. For the first time, we demonstrate that the functional $\mathrm{NAD}^{+}$-binding site in GAPDH is essential for its intranuclear interactions with other macromolecules. Our results suggest a new role for the Rossmann fold in interactions between GAPDH and intranuclear biomolecules.

\section{Materials and Methods}

Cell cultures, drug treatment and plasmids. Colorectal adenocarcinoma SW48-297 (p53 ${ }^{+/+}$) cells were the generous gift from Dr. Vogelstein (John Hopkins University, Baltimore, MR). Cells were maintained in McCoy's A5 medium (Lonza, Allendale, NJ) at $40-80 \%$ confluence. Human GAPDH cDNA was inserted in frame with EGFP into pcDNA3.1 as described earlier (Phadke et al., 2009). Site-mutagenesis of GAPDH ORF was performed using QuickChange Lightning SiteDirected Mutagenesis kit (Stratagene, CA).

Preparation of recombinant human GAPDH. Wild type and variant GAPDH polypeptides with Y94A, S98A, T99A and T99I mutations were cloned into pET28a plasmid (Novagen, Madison, WI). Expressed in BL21 (DE) strain (Novagen, Madison, WI) and purified using Ni-NTA agarose. Fractions with purified GAPDH were pooled, dialyzed against $20 \mathrm{mM}$ TrisHCl $\mathrm{pH}$ 7.9, $50 \mathrm{mM} \mathrm{NaCl}$ and concentrated by ultrafiltration, as described earlier (Phadke et al., 2015). Hisx6 tag was removed by thrombin digestion using Thrombin Cleavage Capture kit (Novagen, Madison, WI) and GAPDH polypeptides were purified by Fast Protein Liquid Chromatography (FPLC) on UNOQ1 anion exchange column $7 \times 35$ (BioRad, Hercules, CA).

RNA Electrophoretic Mobility Shift Assay (REMSA). RNA probe r(AUUUAUUUAUUUAUUUA) labeled at 5'-end with DY682 fluorophore was synthesized by Eurofins MWG Operon (Huntsville, AL). REMSA experiments were performed in $20 \mu \mathrm{L}$ solution containing $10 \mathrm{mM}$ Tris $\mathrm{HCl} \mathrm{pH} 7.5,50 \mathrm{mM} \mathrm{KCl}, 3.5$ mM DTT, poly $\left(\mathrm{dI}^{*} \mathrm{dC}\right) 50 \mathrm{ng} \mu \mathrm{L}^{-1}, 0.05 \% \mathrm{NP}-40,0$ $1000 \mathrm{nM}$ probe and $7.5 \mathrm{ng} \mu \mathrm{L}^{-1}$ protein using Odyssey Infrared EMSA Kit (LI-COR, NE). Reaction mix was incubated for $30 \mathrm{~min}$ at room temperature and the samples were loaded onto a Novex TBE minigel 4-12\% 12 well (Life Technologies, Frederick, MD) and run in $0.5 \times \mathrm{TBE}, 100 \mathrm{~V}$ at $4^{\circ} \mathrm{C}$ for $1 \mathrm{~h}$. The gels were quantified by Odyssey Infrared Imaging system (LICOR Biosciences, Lincoln, NE) using fluorescence detection at $700 \mathrm{~nm}$.

Transfection with EGFP-GAPDH and Fluorescence Recovery after Photobleaching (FRAP) experiments. About 25,000 cells per dish were seeded in $35 \mathrm{~mm}$ glass bottom Petri dishes (MatTek, MA) and transfected with pEGFP-GAPDH plasmid by electroporation (Neon Transfection System, Invitrogen, CA). The next day, cells were treated with $1 \mu \mathrm{M}$ araC for $24 \mathrm{~h}$. FRAP experiments were performed on a Leica TCS SP2 AOBS confocal microscope equipped with a $63 \times / 1.4$ N.A. oil immersion objective at $37^{\circ} \mathrm{C}$, as described earlier (Phadke et al., 2009). Quantitative analysis was performed as described by Rabut and Ellenberg (2005). Diffusion coefficient $D$ value was calculated according to (Axelrod et al., 1976) using the equation $D=0.88^{*} \mathrm{w} 2 /(4 \mathrm{t} 1 / 2)$ where $\mathrm{w}$ is a radius of bleached area, with the assumptions that the bleached area is a disc and that diffusion occurs only laterally (Kappel and Eils, 2004). The immobile fraction was calculated after correction for loss of signal due to photo bleaching (Rabut and Ellenberg, 2005).

Statistical analysis. The statistical significance was determined using Student's t test with Statistica 7.1 software (StatSoft, OK), or one-way analysis of variance with Dunnett's post hoc test, as appropriate and nonlinear regression analysis with GraphPad Prism 4.0 software (GraphPad software, CA). A P value $<0.05$ was considered statistically significant. Data are presented as the mean \pm SD. 


\section{Results}

Mutations in NAD Binding Domain Affect Nuclear Accumulation of EGFP-GAPDH in p53-Proficient Carcinoma Cells

Our molecular modeling experiments predicted T99I to have a strong effect on $\mathrm{NAD}^{+}$binding and catalytic activity of GAPDH (Phadke et al., 2015). In this study we compared GAPDH distribution between cytoplasmic and nuclear compartments in adenocarcinoma cells SW48-297 (p53 $\left.{ }^{+/+}\right)$cells expressing wild type EGFP-GAPDH, or EGFP-T99I fusion protein.

To assess the effect of mutations in the $\mathrm{NAD}^{+}$ binding center on intracellular localization of GAPDH, we used confocal microscopy and image analysis.
Transfected SW48-297 $\left(\mathrm{p} 53^{+/ /}\right)$cells were treated with 1 $\mu \mathrm{M}$ araC for $24 \mathrm{~h}(\mathrm{IC} 50=0.09 \pm 0.019 \mu \mathrm{M})$. After araC treatment, wild type EGFP-GAPDH, as well as EGFPT99Ifusion protein accumulated in the nuclei of SW48$297\left(\mathrm{p} 53^{+++}\right)$cells. Figure 1A shows the distribution of wild type EGFP-GAPDH and EGFP-T99I forms in SW48-297 cells. Wild type and T99I variant demonstrated different intracellular distribution before and after araC treatment: wild type EGFP-GAPDH fusion protein localized in the cytoplasm of untreated cells and accumulated in the nucleus following araC treatment $(11.8 \pm 2.72 \%$ vs. $27.4 \pm 4.28 \%$ nuclear EGFPGAPDH, $\%$ of total EGFP-GAPDH, $p=0.0007)$. T99I EGFP-GAPDH accumulated at high level in the nuclei of untreated and araC-treated cells $(34.3 \pm 8.49 \%$ vs. $41.3 \pm 16.0 \%$ nuclear EGFP-GAPDH, $\%$ of total EGFPGAPDH, $\mathrm{p}=0.21)($ Fig. 1A and B).
A.
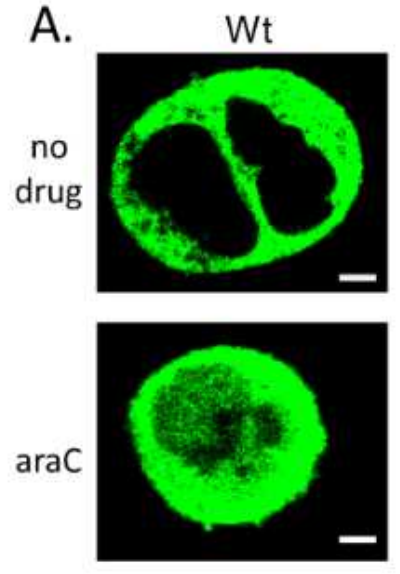

C.

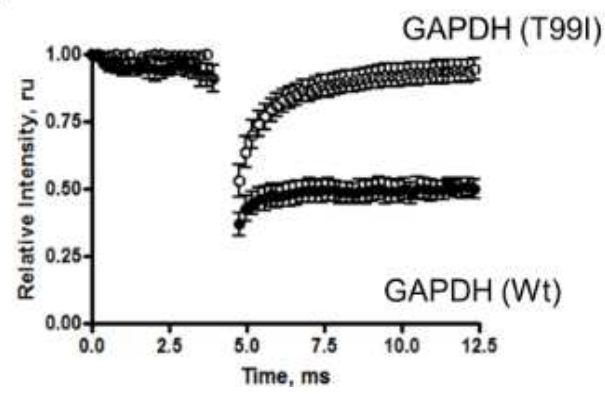

B.
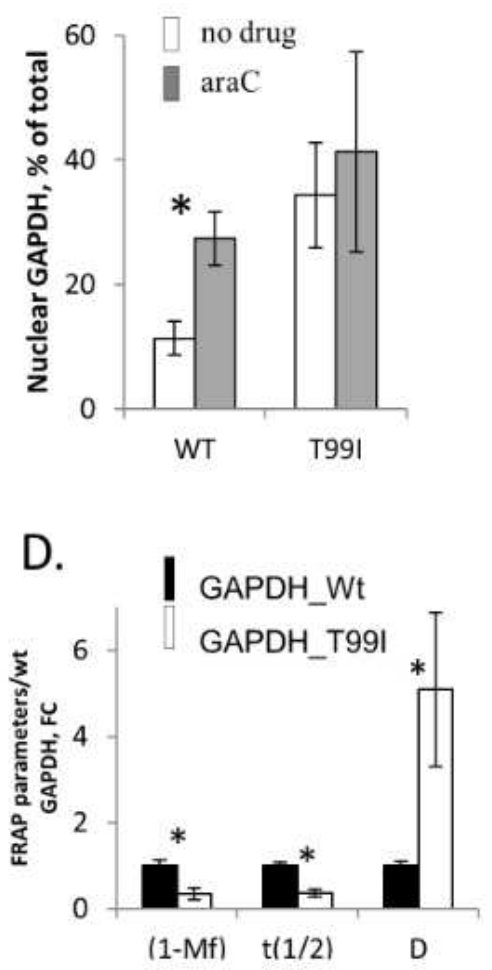

Fig. 1. Mutation within the Rossmann fold changes nuclear GAPDH mobility in SW48-297 cells. Panel A: Confocal images of nuclear accumulation of wild type EGFP-GAPDH and EGFP-T99I (mutated variant) in live SW48-297 cells after transient transfections with plasmids coding for wild type EGFP-GAPDH, or EGFP-T99I before (upper images) and after (lower images) treatment with $1 \mu \mathrm{M}$ araC for $24 \mathrm{~h}$. Scale bar, $10 \mu \mathrm{m}$. Panel B: Quantitative analysis of images shown in Panel A was performed using Image J $1.48 \mathrm{v}$ software (NIH, USA). For statistical evaluation, pixel analysis of 20 cells was performed for each image (mean $\pm \mathrm{SD}$ ). ${ }^{*}, \mathrm{p}<0.03$. Panel $\mathrm{C}$ : Intranuclear mobility estimated by Fluorescence Recovery After Photo bleaching (FRAP) analysis shows difference in recovery rate of wild type EGFP-GAPDH and EGFP-T99I fusion proteins after photo bleaching. Open circles, nuclear EGFP-T99I and black circles, nuclear wild type EGFPGAPDH. Panel D: The dynamic parameters mobile fraction (1-Mf), half-time of equilibration $\mathrm{t}_{(1 / 2)}(\mathrm{s})$ and diffusion coefficients $D$ were calculated from FRAP experiments, as described in "Materials and Methods". Black bars, wild type EGFP-GAPDH and open bars, EGFP-T99I.*, p $<0.05$ 


\section{Mutations within the Rossmann Fold Affect Intranuclear Dynamics of GAPDH}

Recovery of fluorescence intensity in the cytosolic and nuclear compartments was measured by FRAP analysis, as described in "Materials and Methods". Because wild type GAPDH manifests mostly cytoplasmic localization in unstressed cells, its intranuclear level is too low to perform accurate measurements. Therefore, FRAP experiments were performed with the cells pre-treated with araC. Figure 1C shows that recovery of the fluorescent signal was notably different for the wild type and T99I variant EGFP-GAPDH fusion polypeptides. After araC treatment, immobile fraction (1-Mf) of wild type EGFP-GAPDH in the nuclei of SW48-297 cells was about three times higher $(0.75 \pm 0.127$ vs. $0.26 \pm 0.133 \%$, $\mathrm{p}<0.0001$ ), recovery half-time $\mathrm{t}_{1 / 2}$ was about three times higher $(0.84 \pm 0.075$ vs. $0.3 \pm 0.085 \mathrm{~s}, \mathrm{p}<0.0001)$ and diffusion coefficient $D$ was about five times lower (4.6 \pm 0.85 vs. $\left.23.3 \pm 13.79 \mu \mathrm{m}^{2} / \mathrm{s}, \mathrm{p}=0.0001\right)$ compared to cells expressing T99I variant (Fig. 1D).
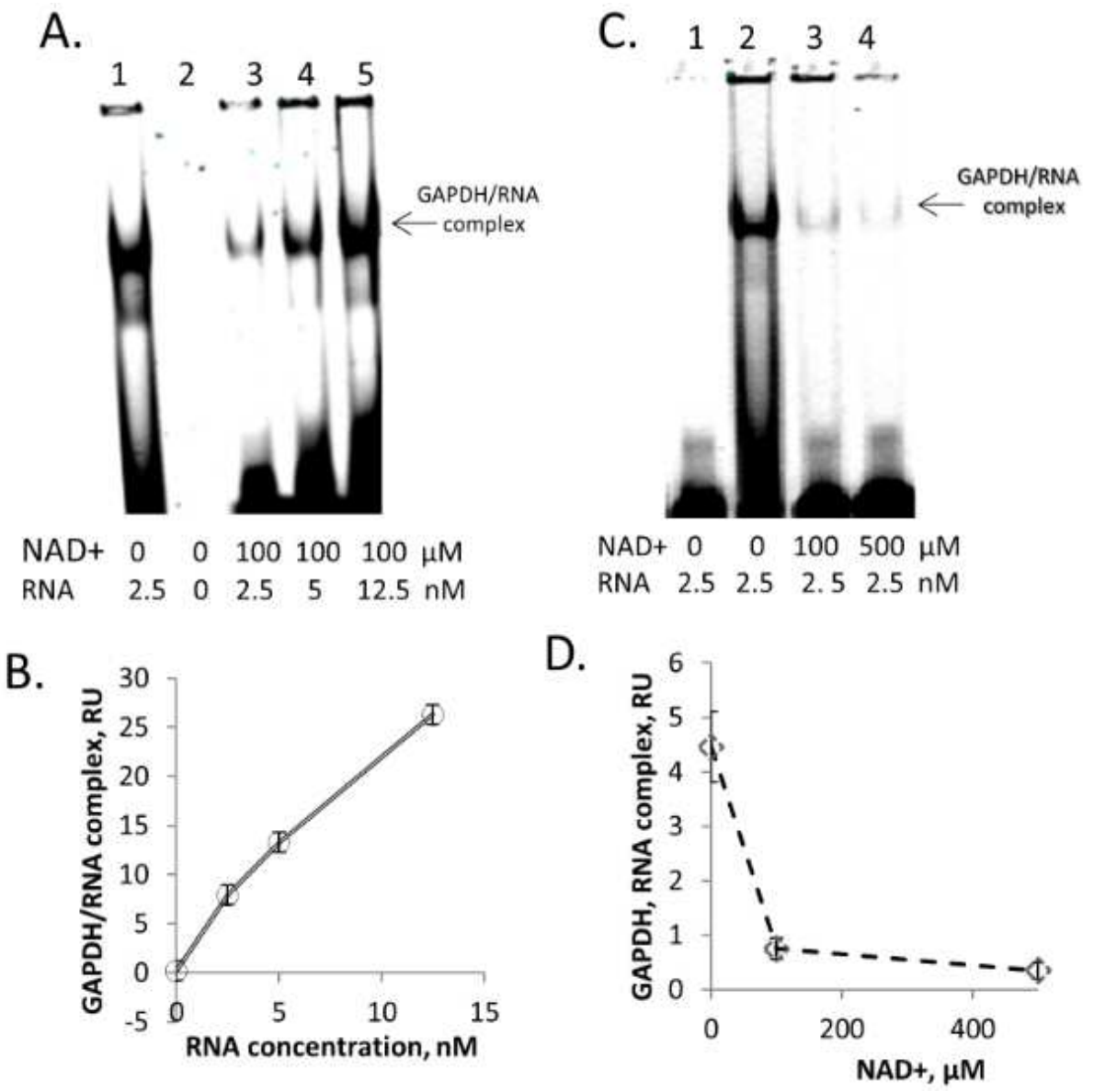

Fig. 2. Inhibition of GAPDH binding to AU-rich RNA by NAD ${ }^{+}$. Panel A: REMSA analysis of RNA/GAPDH complex containing $\mathrm{r}$ (AUUUAUUUAUUUAUUUA) labeled at 5'-end with DY682 fluorophore and $52 \mathrm{nM}$ GAPDH in the presence of increasing concentrations of RNA $(0-12.5 \mathrm{nM})$ and $\mathrm{NAD}^{+}(0$ and $100 \mu \mathrm{M})$. Analysis was performed in 4$12 \%$ Novex TBE minigel $\left(0.5 \times \mathrm{TBE}, 100 \mathrm{~V}\right.$ at $4{ }^{\circ} \mathrm{C}$ for $\left.1 \mathrm{~h}\right)$. Lane $1-$ no NAD ${ }^{+}$added; Lane 2- no RNA added; Lanes 35- increasing concentration of RNA as indicated. Panel B: Quantitative analysis of RNA/GAPDH complex in the presence of increasing RNA concentrations. For quantitative measurement of bound oligonucleotide, fluorescence at $700 \mathrm{~nm}$ was quantified in the gels with the Odyssey Infrared Imaging system as described in "Materials and Methods". The chart shows the results of three independent experiments (mean \pm SD). Panel C: REMSA analysis of the reaction mix containing $12.5 \mathrm{nM}$ r(AUUUAUUUAUUUAUUUA) labeled at 5'-end with DY682 fluorophore and $52 \mathrm{nM}$ GAPDH in the presence of increasing concentrations NAD $(0-500 \mu \mathrm{M})$. Analysis was performed in $4-12 \%$ Novex TBE minigel $\left(0.5 \times \mathrm{TBE}, 100 \mathrm{~V}\right.$ at $4^{\circ} \mathrm{C}$ for $\left.1 \mathrm{~h}\right)$. Lane 1 - no GAPDH added; lanes 2-4- increasing concentration of $\mathrm{NAD}^{+}(0-500 \mu \mathrm{M})$. Panel D: Quantitative analysis of RNA/GAPDH complex in the presence of increasing $\mathrm{NAD}^{+}$ concentrations. The results represent three independent experiments (mean $\pm \mathrm{SD}$ ). Where not seen, error bars are smaller than the data point symbols 


\section{Non-Radioactive RNA Electrophoretic Mobility Shift Assay}

We used a non-radioactive REMSA method based on GAPDH binding to fluorophore-labeled synthetic RNA. 5'terminus of the synthetic probe was conjugated with DY682 reporter group and the sequence of the probe contained 5'-r(AUUUAUUUAUUUAUUUA)-3' tandem repeat identified by Nagy and Rigby as a specific binding site for GAPDH (Nagy and Rigby, 1995). In our preliminary experiments, we found that Hisx6 tag used for affinity purification of GAPDH polypeptides interfered with gel-electrophoresis under REMSA conditions and dramatically decreased GAPDH mobility in non-denaturing gels (not shown). Therefore, recombinant Hisx6-tagged
GAPDH was digested with thrombin and purified by anionexchange FPLC before REMSA experiments. Our method detected formation of RNA-GAPDH complex up to 0.1 fmol fluorophore per band (Fig. 2A and C). REMSA conditions were optimized by titrating RNA in the range 2.5-12.5 nM and $\mathrm{NAD}^{+}$concentrations in the range 0-500 $\mu \mathrm{M}$. Increasing RNA concentration from 2.5 to $12.5 \mathrm{nM}$ led to 3-fold increased RNA-GAPDH complex formation (Fig. 2A and B). In subsequent experiments, we routinely used $2.5 \mathrm{nM}$ RNA. Formation of the RNA-GAPDH complex was inhibited by $\mathrm{NAD}^{+}$(Fig. $2 \mathrm{C}$ and D). In the presence of $100 \mu \mathrm{M} \mathrm{NAD}^{+}$, formation of RNA-GAPDH complex was reduced 5-fold, and $500 \mu \mathrm{M} \mathrm{NAD}^{+}$ completely inhibited complex formation.
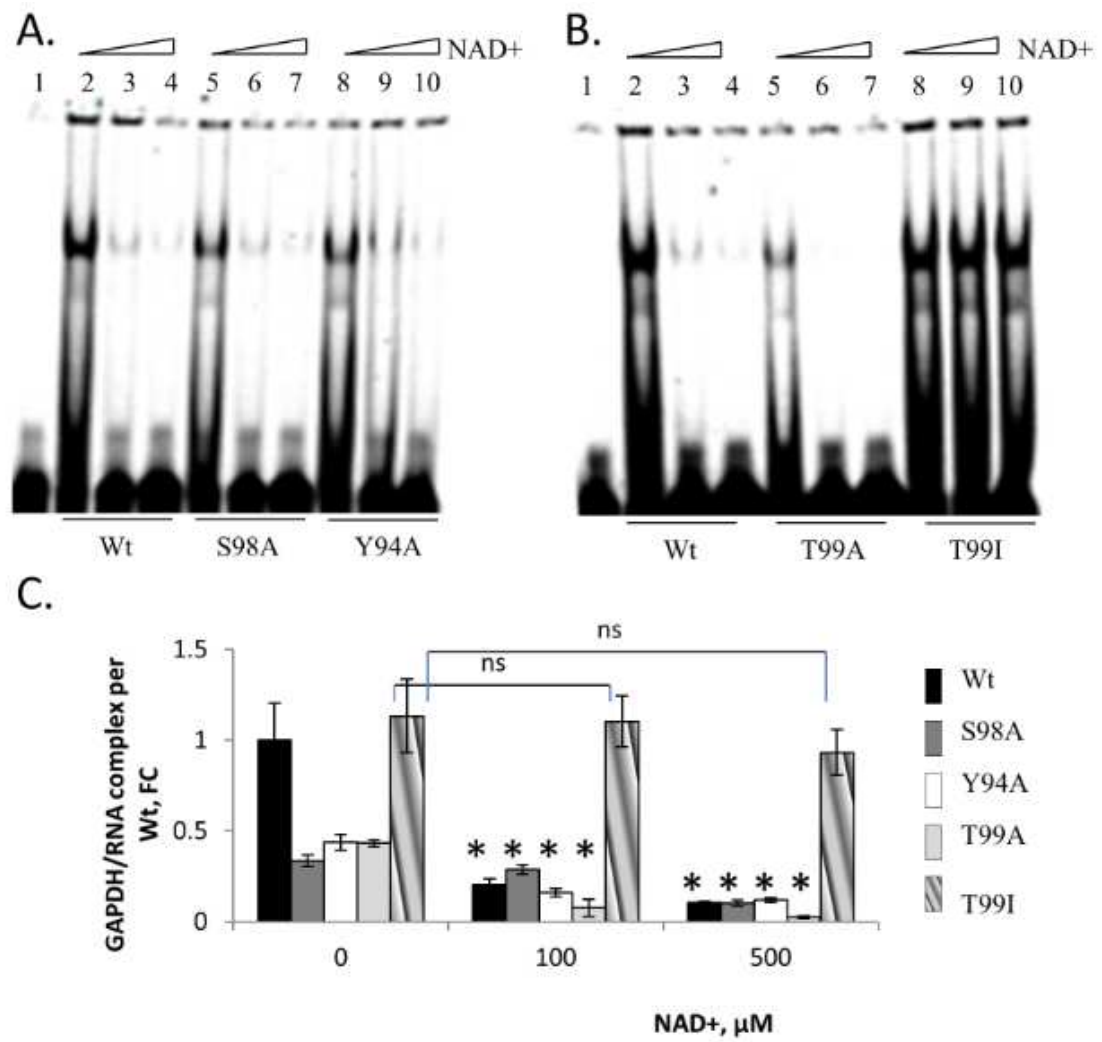

Fig. 3. Mutation at T99I position within the Rossmann fold prevents dissociation of RNA/GAPDH complex in the presence of high $\mathrm{NAD}^{+}$concentrations. Panel A: REMSA analysis of RNA/GAPDH complex containing $12.5 \mathrm{nM}$ r(AUUUAUUUAUUUAUUUA) labeled at 5'-end with DY682 fluorophore and $52 \mathrm{nM}$ either wild type GAPDH, or S98A and Y94A mutated GAPDH variants in the presence of increasing NAD ${ }^{+}$concentration $(0-500 \mu \mathrm{M})$. Analysis was performed in $4-12 \%$ Novex TBE minigel $\left(0.5 \times\right.$ TBE, $100 \mathrm{~V}$ at $4^{\circ} \mathrm{C}$ for $\left.1 \mathrm{~h}\right)$. Lane 1- no GAPDH added; lanes 2-4- wild type GAPDH; lanes 5-7-S98A and lanes 8-10- Y94A with increasing concentration of $\mathrm{NAD}^{+}(0-500 \mu \mathrm{M})$ as indicated. Panel B: REMSA analysis of RNA/GAPDH complex containing $12.5 \mathrm{nM} \mathrm{r}$ (AUUUAUUUAUUUAUUUA) labeled at 5'-end with DY682 fluorophore and $52 \mathrm{nM}$ either wild type GAPDH, or T99A and T99I mutant GAPDH forms in the presence of NAD increasing concentrations $(0-500 \mu \mathrm{M})$. Analysis was performed in $4-12 \%$ Novex TBE minigel $\left(0.5 \times \mathrm{TBE}, 100 \mathrm{~V}\right.$ at $4{ }^{\circ} \mathrm{C}$ for 1 h). Lane 1- no GAPDH added; lanes 2-4- wild type GAPDH; lanes 5-7- T99A and lanes 8-10- T99I with increasing concentrations of $\mathrm{NAD}^{+}(0-500 \mu \mathrm{M})$ as indicated. Panel C: Quantitative analysis of RNA/GAPDH complex formations in wild type GAPDH and its mutated variants in the presence of increasing $\mathrm{NAD}^{+}$concentrations. For quantitative measurement of bound oligonucleotide, fluorescence at $700 \mathrm{~nm}$ was quantified in the gels by Odyssey Infrared Imaging system as described in "Materials and Methods". The results represent three independence experiments (mean \pm SD).ns, not significant. *, $p<0.05$ 


\section{Wild Type and Variant GAPDH Polypeptides Differently Bind to RNA}

We prepared a series of GAPDH polypeptides which contained mutations Y94A, S98A, T99A and T99I within a peptide WGDAGAEYVVESTGVFTTMEK corresponding to the amino acid sequence 87-107 of human GAPDH, an element of the $\mathrm{NAD}^{+}$binding center in GAPDH polypeptide. Using REMSA, we compared RNA-binding properties of wild type and variant GAPDH polypeptides in the presence of increasing $\mathrm{NAD}^{+}$concentrations (Fig. 3A and B). Quantification of REMSA gel images revealed that amino acid change at positions 94,98 , or 99 to alanine resulted in two-fold decrease of RNA binding to GAPDH (Fig. 3C). Addition of 100-500 $\mu \mathrm{M} \mathrm{NAD}{ }^{+}$caused 5 to 9-fold decrease of RNA binding to wild type GAPDH. Similarly, RNA binding to Y94A, S98A and T99A variants was inhibited by $50-95 \%$ in the presence of $\mathrm{NAD}^{+}$(Fig. 3C). In contrast, GAPDH variant T99I demonstrated RNA binding activity comparable to that of wild type GAPDH $(\mathrm{p}=0.5651)$. Increased $\mathrm{NAD}^{+}$concentration did not affect T99I-RNA binding (Fig. 3B and C).

\section{Discussion}

Endogenous GAPDH and ectopically expressed EGFP-GAPDH fusion polypeptides localize predominantly in the cytoplasm of unstressed A549, SW620, DLD1 and HCT116-40 cells and accumulate in the nucleus after various stress stimuli (Brown et al., 2004; Phadke et al., 2009; 2015). In the nuclei of the stressed cells, GAPDH tightly binds to chromatin (Sawa et al., 1997; Lenglet et al., 2013). FRAP experiments revealed reduced mobility of nuclear GAPDH, presumably due to binding to unidentified nuclear components (Phadke et al., 2009).

Our experiments revealed that mutation of phosphoamino acids Y94, S98 and T99 affected glycolytic activity, distribution and intranuclear interactions of GAPDH. Because all mutations were introduced into the same region of a polypeptide containing the Rossmann fold (Buehner et al., 1973), it is conceivable that the above functions of GAPDH are interrelated.

Phosphoamino acids Y94, S98 and T99 are components of the $\mathrm{NAD}^{+}$binding center in GAPDH and are likely to form an RNA-binding center (Nagy and Rigby, 1995; Jenkins and Tanner, 2006). Our previous results demonstrated that the change of these amino acids to non-phosphorylated alanine or isoleucine disrupted the cofactor binding site thus inhibiting GAPDH glycolytic activity. In our site-directed mutagenesis experiments, we performed alanine scanning where amino acid residues of interest were substituted by alanine. Alanine does not introduce a phosphorylation site, charge, steric restrictions, or other strong structural effects to the peptide chain. In addition, we prepared a T99I variant of GAPDH. Thr->Ile change at position 99 results from a single nucleotide variation rs11549329 found in NCBI dbSNP database. We did not assess Ile at positions 94 or 98, as no similar mutant GAPDH variants had been found in clinical samples. In vitro experiments demonstrated that among four mutated GAPDH variants, T99I variant manifested the lowest affinity to $\mathrm{NAD}^{+}$and nondetectable glycolytic activity (Phadke et al., 2015).

Y94A, S98A and T99A variants show reduced RNA binding compared to wild type GAPDH. High $\mathrm{NAD}^{+}$ concentration (100-500 $\mu \mathrm{M})$ inhibits formation of RNAGAPDH complexes with Y94A, S98A and T99A variants. These findings support the premise that RNA binds to Y94A, S98A and T99A variants at the NAD ${ }^{+}$binding center. In contrast, in the absence of $\mathrm{NAD}^{+}$the fraction of T99I bound to RNA was comparable to that of wild type GAPDH. $\mathrm{NAD}^{+}$concentration up to 500 $\mu \mathrm{M}$ did not inhibit T99I binding to RNA (Fig. 3B and D). This observation indicates that RNA binds to a different site on T99I protein. $\mathrm{NAD}^{+}$concentration in human cell nuclei is about $100 \mu \mathrm{M}$ (Koch-Nolte et al., 2011). Therefore, wild type GAPDH is likely bound to $\mathrm{NAD}^{+}$rather than $\mathrm{RNA}$, while T99I variant remains bound to RNA at this $\mathrm{NAD}^{+}$concentration. A recent study suggests a novel mechanism for regulation of GAPDH glycolytic activity via depletion of $\mathrm{NAD}^{+}$ caused by PARP activation (Yun et al., 2015). A functional switch between $\mathrm{NAD}^{+}$-bound GAPDH and RNA-bound GAPDH could provide a hypothetical mechanism for $\mathrm{NAD}^{+}$delivery to $\mathrm{NAD}^{+}$-dependent proteins such as PARP1 or sirtuins.

Two fusion proteins, wild type EGFP-GAPDH and EGFP-T99I variant are distributed differently between cytoplasmic and nuclear compartments in untreated cells, as well as after araC treatment. Significantly higher fraction of EGFP-T99I variant was detected in the nuclear compartment of untreated SW48-297 cells, compared to EGFP-GAPDH (Fig. 1A and B). After araC treatment, the fraction of nuclear wild type EGFPGAPDH increased two times, but there was no significant change in the level of nuclear EGFP-T99I (Fig. $1 \mathrm{~A}$ and $\mathrm{B})$. It remains to be explored if predominantly nuclear localization of T99I variant results from formation of a stable T99I GAPDH-RNA complex which subsists even at high $\mathrm{NAD}^{+}$concentration.

Molecular dynamics parameters (diffusion coefficient $D$ and immobile fraction $\left(1-\mathrm{M}_{\mathrm{f}}\right)$ ) of fusion polypeptides EGFP-GAPDH and EGFP-T99I variant in SW48-297 cells treated with araC were also found to be quite different. Diffusion coefficient $D$ value for wild type EGFP-GAPDH in the nuclei of SW48-297 cells was significantly lower than that of cytoplasmic EGFP- 
GAPDH. Moreover, a high proportion of EGFP-GAPDH was immobilized inside the nuclei of SW48-297 cells, as estimated by $\left(1-\mathrm{M}_{\mathrm{f}}\right)$ value. Both findings indicate that, inside the nucleus, wild type EGFP-GAPDH is tightly bound to yet unknown macromolecular components. With EGFP-T99I variant, diffusion coefficient $D$ was about five times higher and immobile fraction was about three times lower than the values for the wild type EGFP-GAPDH. These results are in line with our findings in HCT116-40 cultured cells (Phadke et al., 2015). We hypothesize that the T99I variant fails to recruit intranuclear components because of its nonfunctional $\mathrm{NAD}^{+}$-binding center. We speculate that $\mathrm{NAD}^{+}$binding is a pre-requisite for GAPDH intranuclear interaction with yet to be identified nuclear macromolecules.

The biochemical significance of GAPDH interaction with RNA is poorly understood. GAPDH-RNA binding alters mRNA stability (Bonafe et al., 2005; Ikeda et al., 2012; Zeng et al., 2014). Several reports suggest that GAPDH binding to RNA may play a role in nucleocytoplasmic transport of RNA (Singh and Green, 1993; Muller et al., 1992; Hamilton et al., 1993). Alternatively, GAPDH-RNA binding may change intracellular localization of GAPDH, a process potentially regulated by local $\mathrm{NAD}^{+}$concentration.

Our experiments showed that wild type EGFPGAPDH (apparently bound to $\mathrm{NAD}^{+}$) manifested decreased intranuclear mobility indicative of tight GAPDH binding with unknown entities in the nucleus. On the other hand, T99I mutant protein with distorted $\mathrm{NAD}^{+}$binding center and abrogated $\mathrm{NAD}^{+}$binding retains RNA-binding properties and manifests high intranuclear mobility. Taken together, our results do not support the hypothesis that immobilization of nuclear GAPDH occurs due to formation of GAPDH-RNA complex. Interestingly, treatment of nuclei with DNase/RNase/2 M salt did not mobilize GAPDH suggesting that GAPDH inside the nucleus might be involved in interactions with nuclear proteins (Berry, 2004).

Formation of binary GAPDH-RNA complexes could be only a part of the story, as several multiprotein complexes containing both GAPDH and RNA have been detected and characterized. Involvement of GAPDH in regulated activation of gene transcription via formation of ER- $\alpha$-ERE complex was recently demonstrated (Velazquez-Villegas et al., 2014). Another component of the same complex is $\mathrm{NAD}^{+}$-dependent polymerase PARP-1, which works in concert with HMGB1 in signal-regulated transcription ( Ju et al., 2006). Intriguingly, GAPDH was detected as a component of HMGB1-containing multiprotein complex involved in DNA damage repair (Krynetski et al., 2001; 2003). This multiprotein complex dissociated upon RNase treatment suggesting RNA as one of the structural elements (Krynetskiy, unpublished). Several other GAPDH/RNA- containing multiprotein complexes were shown to be sensitive to RNase treatment and to dissociate upon RNA hydrolysis (Mazurek et al., 1996; Carlile et al., 1998). Taken together, these observations suggest that the RNA-GAPDH may provide a surface for mounting other proteins during the process of complex formation. If proved true, this could explain co-purification of GAPDH with many biochemically unrelated macromolecules which led to the idea of GAPDH as a moonlighting protein.

\section{Conclusion}

Our experiments demonstrated that T99I mutation disrupts $\mathrm{NAD}^{+}$binding but not RNA binding indicating that RNA interacts with a different site in T99I protein. In the wild type protein with a functional $\mathrm{NAD}^{+}$binding center, the switch between GAPDH-NAD ${ }^{+}$and GAPDHRNA binding could provide a mechanism by which $\mathrm{NAD}^{+}$is supplied to other components of the GAPDHcontaining complex. Inside the nucleus, the wild type EGFP-GAPDH manifests molecular dynamics parameters characteristic of tight binding with macromolecules, presumably nuclear proteins. T99I mutation abrogates binding to nuclear components and immobilization of EGFP-GAPDH inside the nucleus. Our study indicates that the functional $\mathrm{NAD}^{+}$-binding site in GAPDH is essential for intranuclear interactions with other macromolecules and suggests the functional role of GAPDH as a scaffold for $\mathrm{NAD}^{+}$-dependent multiprotein complexes.

\section{Acknowledgement}

We gratefully acknowledge Dr. Anurag Mishra for preparing GAPDH constructs and Nilay Shah for transfection experiments, Dr. Bettina Buttaro and Dr. Patrick Piggot for their help with confocal microscopy and FRAP experiments.

\section{Funding Information}

This work was supported by the Temple University Summer Research Grant (to EK) and the Jayne Haines Center for Pharmacogenomics and Drug Safety at Temple University.

\section{Author's Contributions}

Natalia Krynetskaia: Designed the study, developed experimental protocols and drafted and revised the paper.

Manali Phadke: Performed most of the experiments.

Evgeny Krynetskiy: Designed the study, developed experimental protocols, evaluated the results and participated in manuscript preparation. 


\section{Ethics}

This article is original and contains unpublished material. All co-authors have read and approved the manuscript. All co-authors of the manuscript have no conflict of interest and have nothing to disclose.

\section{References}

Axelrod, D., D.E. Koppel, J. Schlessinger, E. Elson and W.W. Webb, 1976. Mobility measurement by analysis of fluorescence photobleaching recovery kinetics. Biophys. J., 16: 1055-1069.

DOI: $10.1016 / \mathrm{S} 0006-3495(76) 85755-4$

Backlund, M., K. Paukku, L. Daviet, R.A. De Boer and E. Valo et al., 2009. Posttranscriptional regulation of angiotensin II type 1 receptor expression by glyceraldehyde 3-phosphate dehydrogenase. Nucleic Acids Res., 37: 2346-2358. DOI: 10.1093/nar/gkp098

Berry, M.D., 2004. Glyceraldehyde-3-phosphate dehydrogenase as a target for small-molecule disease-modifying therapies in human neurodegenerative disorders. J. Psychiatry Neurosci., 29: 337-345. PMID: 15486605

Bonafe, N., M. Gilmore-Hebert, N.L. Folk, M. Azodi and Y. Zhou et al. 2005. Glyceraldehyde-3phosphate dehydrogenase binds to the AU-Rich 3' untranslated region of colony-stimulating factor-1 (CSF-1) messenger RNA in human ovarian cancer cells: possible role in CSF-1 posttranscriptional regulation and tumor phenotype. Cancer Res., 65: 3762-3771. DOI: 10.1158/0008-5472.CAN-04-3954

Brown, V.M., E.Y. Krynetski, N.F. Krynetskaia, D. Grieger and S.T. Mukatira et al., 2004. A novel CRM1mediated nuclear export signal governs nuclear accumulation of glyceraldehyde-3-phosphate dehydrogenase following genotoxic stress. J. Biol. Chem., 279: 5984-5992.

DOI: $10.1074 /$ jbc.M307071200

Buehner, M., G.C. Ford, D. Moras, K.W. Olsen and M.G. Rossmann, 1973. D-Glyceraldehyde-3phosphate dehydrogenase: Three-dimensional structure and evolutionary significance. Proc. Nat. Acad. Sci. USA, 70: 3052-3054.

DOI: $10.1073 /$ pnas.70.11.3052

Carlile, G.W., W.G. Tatton and K.L. Borden, 1998. Demonstration of a RNA-dependent nuclear interaction between the promyelocytic leukaemia protein and glyceraldehyde-3-phosphate dehydrogenase. Biochem. J., 335: 691-696. DOI: $10.1042 /$ bj3350691

Demarse, N.A., S. Ponnusamy, E.K. Spicer, E. Apohan and J.E. Baatz et al., 2009. Direct binding of glyceraldehyde 3-phosphate dehydrogenase to telomeric DNA protects telomeres against chemotherapy-induced rapid degradation. J. Mol. Biol., 394: 789-803.

DOI: $10.1016 /$ j.jmb.2009.09.062
Hamilton, B.J., E. Nagy, J.S. Malter, B.A. Arrick and W.F. Rigby, 1993. Association of heterogeneous nuclear ribonucleoprotein $\mathrm{A} 1$ and $\mathrm{C}$ proteins with reiterated AUUUA sequences. J. Biol. Chem., 268: 8881-8887. PMID: 8473331

Ikeda, Y., R. Yamaji, K. Irie, N. Kioka and A. Murakami, 2012. Glyceraldehyde-3-phosphate dehydrogenase regulates cyclooxygenase- 2 expression by targeting mRNA stability. Arch. Biochem. Biophys., 528: 141-147. DOI: 10.1016/j.abb.2012.09.004

Jenkins, J.L. and J.J. Tanner, 2006. High-resolution structure of human D-glyceraldehyde-3-phosphate dehydrogenase. Acta Crystallogr., 62: 290-301. DOI: $10.1107 / \mathrm{S} 0907444905042289$

Ju, B.G., V.V. Lunyak, V. Perissi, I. Garcia-Bassets and D.W. Rose et al., 2006. A topoisomerase IIßmediated dsDNA break required for regulated transcription. Science, 312: 1798-1802. DOI: $10.1126 /$ science. 1127196

Kappel, C. and R. Eils, 2004. Fluorescence recovery after photobleaching with Leica TCS SP2. Confocal Appli. Lett.

Koch-Nolte, F., S. Fischer, F. Haag and M. Ziegler, 2011. Compartmentation of $\mathrm{NAD}^{+}$-dependent signalling. FEBS Lett. 585:1651-1656. DOI: $10.1016 /$ j.febslet.2011.03.045

Kondo, S., S. Kubota, Y. Mukudai, T. Nishida and Y. Yoshihama et al., 2011. Binding of glyceraldehyde-3-phosphate dehydrogenase to the cis-acting element of structure-anchored repression in ccn 2 mRNA. Biochem., Biophys. Res. Commun., 405: 382-387. DOI: 10.1016/j.bbrc.2011.01.034

Krynetski, E.Y., N.F. Krynetskaia, M.E. Bianchi and W.E. Evans, 2003. A nuclear protein complex containing high mobility group proteins $\mathrm{B} 1$ and $\mathrm{B} 2$, heat shock cognate protein 70, ERp60 and glyceraldehyde-3-phosphate dehydrogenase is involved in the cytotoxic response to DNA modified by incorporation of anticancer nucleoside analogues. Cancer Res., 63: 100-106. PMID: 12517784

Krynetski, E.Y., N.F. Krynetskaia, A.E. Gallo, K.G. Murti and W.E. Evans, 2001. A novel protein complex distinct from mismatch repair binds thioguanylated DNA. Mol. Pharmacol., 59: 367-374. PMID: 11160874

Lenglet, G., S. Depauw, D. Mendy and M.H. David-Cordonnier, 2013. Protein recognition of the S23906-1-DNA adduct by nuclear proteins: Direct involvement of glyceraldehyde-3-phosphate dehydrogenase (GAPDH). Biochem. J., 452: 147-159. DOI: $10.1042 / \mathrm{BJ} 20120860$

Mazurek, S., F. Hugo, K. Failing and E. Eigenbrodt, 1996. Studies on associations of glycolytic and glutaminolytic enzymes in MCF-7 cells: Role of P36. J. Cell. Physiol., 167: 238-250.

DOI: $10.1002 /(\mathrm{SICI}) 1097-$ 4652(199605)167:2<238::AID-JCP7>3.0.CO;2-Q 
Muller, W.E., H. Slor, K. Pfeifer, P. Huhn and A. Bek et al., 1992. Association of AUUUA-binding protein with A+U-rich mRNA during nucleo-cytoplasmic transport. J. Mol. Biol., 226: 721-733.

DOI: $10.1016 / 0022-2836(92) 90628-\mathrm{W}$

Nagy, E. and W.F. Rigby, 1995. Glyceraldehyde-3phosphate dehydrogenase selectively binds AU-rich RNA in the $\mathrm{NAD}^{+}$-binding region (Rossmann fold). J. Biol. Chem., 270: 2755-2763. DOI: $10.1074 / \mathrm{jbc} .270 .6 .2755$

Phadke, M., N. Krynetskaia, A. Mishra, C. Barrero and S. Merali et al., 2015. Disruption of $\mathrm{NAD}^{+}$binding site in glyceraldehyde 3-phosphate dehydrogenase affects its intranuclear interactions. World J. Biol. Chem., 6: 366-378. DOI: 10.4331/wjbc.v6.i4.366

Phadke, M.S., N.F. Krynetskaia, A.K. Mishra and E. Krynetskiy, 2009. Glyceraldehyde 3-phosphate dehydrogenase depletion induces cell cycle arrest and resistance to antimetabolites in human carcinoma cell lines. J. Pharmacol. Exp. Ther., 331: 77-86. DOI: $10.1124 /$ jpet.109.155671

Rabut, G. and J. Ellenberg, 2005. Photobleaching techniques to study mobility and molecular dynamics of proteins in live cells: FRAP, iFRAP and FLIP. In: Live Cell Imaging, Goldman, R.D. and D.L. Spector (Eds.), Cold Spring Harbor, NY, pp: 101-126.

Rodriguez-Pascual, F., M. Redondo-Horcajo, N. Magan-Marchal, D. Lagares and A. MartinezRuiz et al., 2008. Glyceraldehyde-3-phosphate dehydrogenase regulates endothelin-1 expression by a novel, redox-sensitive mechanism involving mRNA stability. Mol. Cell. Biol., 28: 7139-7155. DOI: 10.1128/MCB.01145-08

Rossman, M.G., 1981. Evolution of glycolytic enzymes. Philos. Trans. R Socity, 293: 191-203. DOI: $10.1098 /$ rstb.1981.0072

Sawa, A., A.A. Khan, L.D. Hester and S.H. Snyder, 1997. Glyceraldehyde-3-phosphate dehydrogenase: Nuclear translocation participates in neuronal and nonneuronal cell death. Proc. Nat. Acad. Sci. USA, 94: 11669-11674. DOI: 10.1073/pnas.94.21.11669

Seidler, N.W., 2013. Functional diversity. Adv. Exp. Med. Biol., 985: 103-147.
Singh, R. and M.R. Green, 1993. Sequence-specific binding of transfer RNA by glyceraldehyde-3phosphate dehydrogenase. Science, 259: 365-368. DOI: $10.1126 /$ science. 8420004

Sundararaj, K.P., R.E. Wood, S. Ponnusamy, A.M. Salas and Z. Szulc et al., 2004. Rapid shortening of telomere length in response to ceramide involves the inhibition of telomere binding activity of nuclear glyceraldehyde-3-phosphate dehydrogenase. J. Biol. Chem., 279: 6152-6162. DOI: $10.1074 /$ jbc.M310549200

Velazquez-Villegas, L.A., V. Ortiz, A. Strom, N. Torres and D.A. Engler et al., 2014. Transcriptional regulation of the sodium-coupled neutral amino acid transporter (SNAT2) by $17 \beta$-estradiol. Proc. Nat. Acad. Sci. USA, 111: 11443-11448. DOI: $10.1073 /$ pnas.1412099111

White, M.R., M.M. Khan, D. Deredge, C.R. Ross and R. Quintyn et al., 2015. A dimer interface mutation in glyceraldehyde 3-phosphate dehydrogenase regulates its binding to AU-rich RNA. J. Biol. Chem., 290: 4129-4129. DOI: 10.1074/jbc.A114.618165

Yun, J., E. Mullarky, C. Lu, K.N. Bosch and A. Kavalier et al., 2015. Vitamin C selectively kills KRAS and BRAF mutant colorectal cancer cells by targeting GAPDH. Science, 350: 1391-1396. DOI: $10.1126 /$ science.aaa5004

Zeng, T., Z.F. Dong, S.J. Liu, R.P. Wan and L.J. Tang et al., 2014. A novel variant in the 3' UTR of human SCN1A gene from a patient with Dravet syndrome decreases mRNA stability mediated by GAPDH's binding. Hum. Genet., 133: 801-811. DOI: $10.1007 / \mathrm{s} 00439-014-1422-8$

Zhou, Y., X. Yi, J.B. Stoffer, N. Bonafe and M. Gilmore-Hebert et al., 2008. The multifunctional protein glyceraldehyde-3-phosphate dehydrogenase is both regulated and controls colony-stimulating factor-1 messenger RNA stability in ovarian cancer. Mol. Cancer Res., 6: 1375-1384.

DOI: 10.1158/1541-7786.MCR-07-2170 